\author{
MITSUBISHI ELECTRIC RESEARCH LABORATORIES \\ http://www.merl.com
}

\title{
A Non-Coherent 802.15.4a UWB Impulse Radio
}

\author{
Chunjie Duan, Philip Orlik, Zafer Sahinoglu and Andreas F. Molisch
}

TR2007-070 September 2007

\begin{abstract}
The new IEEE 802.15.4a standard for Wireless Personal Area Networks (WPAN) extends the capabilities of its popular predecessor IEEE 802.15.4, and enables accurate range/distance estimation between communicating devices. In addition to its ranging capabilities, the new standard also defines several new data rates ranging from $100 \mathrm{~kb} / \mathrm{s}$ to $27 \mathrm{Mb} / \mathrm{s}$ and a variety of options that give IEEE 802.15.4a compliant devices a high degree of flexibility and scalability. In this article we describe our implementation of an UWB impulse radio device, which is compliant with the 802.15.4a standard. Our implementation uses non-coherent energy-detection based receivers both to demodulate data and to detect ranges. We will present our prototype system and discuss some implementation details (energy detection, sampling rates, acquisition/synchronization) specific to non-coherent receivers. The performance of our prototype system is discussed as well. The reported range estimation error is less than $30 \mathrm{~cm}$, which agrees well with theoretical predictions.
\end{abstract}

IEEE International Conference on Ultra-Wideband (ICUWB)

\footnotetext{
This work may not be copied or reproduced in whole or in part for any commercial purpose. Permission to copy in whole or in part without payment of fee is granted for nonprofit educational and research purposes provided that all such whole or partial copies include the following: a notice that such copying is by permission of Mitsubishi Electric Research Laboratories, Inc.; an acknowledgment of the authors and individual contributions to the work; and all applicable portions of the copyright notice. Copying, reproduction, or republishing for any other purpose shall require a license with payment of fee to Mitsubishi Electric Research Laboratories, Inc. All rights reserved.
} 



\title{
A Non-Coherent 802.15.4a UWB Impulse Radio
}

\author{
(Invited Paper) \\ Chunjie Duan, Philip Orlik, Zafer Sahinoglu and Andreas F. Molisch \\ Mitsubishi Electric Research Labs \\ 201 Broadway, Cambridge, MA 02139, USA \\ Email: \{duan, porlik, zafer, molisch\}@merl.com
}

\begin{abstract}
The new IEEE 802.15.4a standard for Wireless Personal Area Networks (WPAN) extends the capabilities of its popular predecessor IEEE 802.15.4, and enables accurate range/distance estimation between communicating devices. In addition to its ranging capabilities, the new standard also defines several new data rates ranging from $100 \mathrm{~kb} / \mathrm{s}$ to $27 \mathrm{Mb} / \mathrm{s}$ and a variety of options that give IEEE 802.15.4a compliant devices a high degree of flexibility and scalability. In this article we describe our implementation of an UWB impulse radio device, which is compliant with the 802.15.4a standard. Our implementation uses non-coherent energy-detection based receivers both to demodulate data and to detect ranges. We will present our prototype system and discuss some implementation details (energy detection, sampling rates, acquisition/synchronization) specific to non-coherent receivers. The performance of our prototype system is discussed as well. The reported range estimation error is less than $30 \mathrm{~cm}$, which agrees well with theoretical predictions.
\end{abstract}

\section{INTRODUCTION}

The IEEE 802.15.4a task group has been working on an improved PHY layer based on ultrawideband (UWB) since 2004. The major goals of the standard are "...in providing communications and high precision ranging / location capability (1 meter accuracy and better), high aggregate throughput, and ultra low power; as well as adding scalability to data rates, longer range, and lower power consumption and cost." [1]. These additional capabilities, particularly ranging, are expected to enable significant new applications and market opportunities. As this paper is being written, the final draft standard has been completed and is expected to be published within the next few months.

The standard is crafted to allow the coexistence of different types of devices: coherent and non-coherent in the same network. Coherent-receiver based devices offer longer range and better BER performance in noisy channel condition. However, these type of devices generally have higher complexity (hence high cost) and power consumption. In many applications where cost and energy consumption are critical, non-coherent devices are desirable even though they may have shorter communication range and inferior performance under certain channel conditions.

Even though a non-coherent radio is simpler than a coherent receiver, it is still challenging to realize a low complexity and low power implementation due to the wide signal bandwidth and the requirements imposed by ranging. In this paper, we present our implementation of a non-coherent $802.15 .4 \mathrm{a}$ radio.
Using the prototype radio, we demonstrated data communication and ranging with better than $30 \mathrm{~cm}$ accuracy. We also demonstrated networking capability using the prototype radios and achieved high precision positioning.

The paper is organized as follows: Section II describes basic features in the standard in both the data communication and ranging; In Section III we will discuss issues on implementing a low complexity, power efficient non-coherent node with high ranging accuracy based on the prototype we developed; In Section IV we will show some experimental results of our prototype system. In Section V we draw the conclusion.

\section{BASIC FEATURES OF 802.15.4A STANDARD}

The 802.15.4a standard uses a UWB physical layer to enable data communications and precision ranging ${ }^{1}$. A major objective of the standard is support for both coherent and non-coherent receivers within the same network; the motivation being that not all devices in a network have the same application and cost requirements. For example, a wireless sensor networks for building management may have a need simple low cost sensors to measure temperature or occupancy. Selected nodes in the same network may need to provide critical functions such as fire alarms, which require that these devices be capable of communicating at longer range and with more robustness to interference and multipath while power efficiency is not as large a concern. The remainder of this section describes the most salient features of the $802.15 .4 \mathrm{a}$ standard that enable this. such support. A more extensive tutorial can be found in [3].

\section{A. Data Communication}

The 802.15.4a standard uses a combination of burst position modulation (BPM) and binary phase shift keying (BPSK) to support both coherent and non-coherent receivers using a common signaling scheme. The combined BPM-BPSK is used to modulate symbols, with each symbol being composed of a burst of UWB pulses. The position of this burst within the first half or second half of a data symbol period, $T_{\text {sym }}$, indicates a bit of ' 0 ' or '1' respectively. Additionally, the burst polarity $(+1$ or -1$)$ is used to modulate a second information bit. The polarity modulation, however, is used to convey a redundant bit (created by a convolutional code) rather than user data, since non-coherent receivers are incapable of recovering the pulse

\footnotetext{
${ }^{1}$ it also defines a Chirp Spread Spectrum (CSS) mode, which is outside the scope of this paper
} 


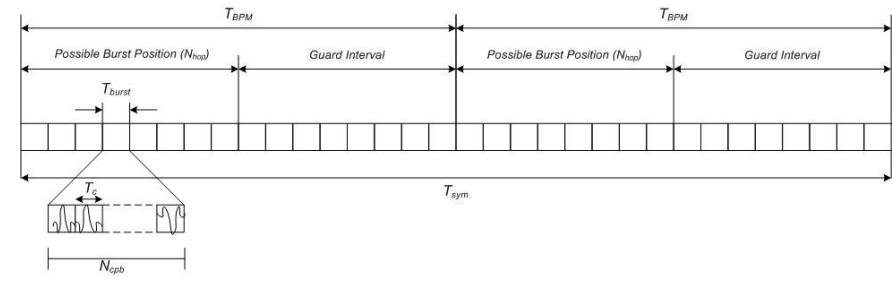

Fig. 1. IEEE 802.15.4a Data Symbol Structure

burst phase. Details of the forward error correction (FEC) used to generate the parity check bits can be found in [2] and are omitted here since they do not impact the non-coherent receiver.

The structure and timing of a UWB PHY symbol is illustrated in Figure 1 Each symbol consists of an integer number of possible chip positions, $N_{c}$, each with duration, $T_{c}=2.003 \mathrm{~ns}$ (which corresponds to a chipping rate of 499.2 $\mathrm{MHz}$ ). The overall symbol duration is given by $T_{\text {sym }}=N_{c} T_{c}$. Furthermore, each symbol is divided into two BPM intervals each with duration $T_{B P M}=T_{\text {sym }} / 2$ which enables the binary position modulation. A burst is formed by grouping $N_{c} p b$ consecutive chips and has duration $T_{\text {burst }}=N_{c p b} T_{c}$. In each UWB PHY symbol interval a single burst event is transmitted. Note also that the burst duration is typically much shorter than the BPM duration, i.e. $T_{\text {burst }}<<T_{B P M}$. This provides for some multi-user access interference rejection in the form of time hopping. The total number of burst durations per symbol, $N_{\text {burst }}$, is given by $N_{\text {burst }}=T_{\text {sym }} / T_{\text {burst }}$. In order to limit the amount of inter-symbol interference caused by multi-path only the first half of each $T_{B P M}$ period contains a burst, therefore, only the first $N_{h o p}=N_{\text {burst }} / 4$ possible burst positions are candidate hopping burst positions within each BPM interval. Each burst position can be varied on a symbol to symbol basis according to a time hopping code.

\section{B. Ranging Capability}

Even though ranging is an optional feature, it is one of the most distinctive features of the 802.15.4a standard. The large bandwidth of the ultrawideband signals enables highly accurate ranging [4]. A specially designed preamble also helps to identify multipath components.

The ranging preamble can consist of $\{16,64,1024,4096\}$ symbols. The longer lengths $\{1024,4096\}$ are preferred for non-coherent receivers to help them improve the signal to noise ratio (SNR) via processing gain. Hence, they can provide a better time-of-arrival estimate. Each of the symbols of the ranging preamble uses one of the length-31 ternary sequences, $S_{i}$, in Table I.

Each one of these sequences has a perfect periodic autocorrelation, as well as perfect (pseudo-) autocorrelation function of the rectified (energy-detected) sequence. Therefore, multipath components in the arriving signal can be easily identified. Assume that $\phi(t)$ is the transmitted UWB pulse waveform with unit energy, $T_{\text {sym }}$ denotes the symbol duration, $N_{\text {sym }}$ is the
TABLE I

THE BASIS PREAMBLE SYMBOL SET

\begin{tabular}{cc}
\hline \hline Index & Symbol \\
\hline$S_{1}$ & $-1000010-1011101-10001-111100-110-100$ \\
$S_{2}$ & $0101-10101000-1110-11-1-1-10010011000$ \\
$S_{3}$ & $-11011000-11-11100110100-10000-1010-1$ \\
$S_{4}$ & $00001-100-100-1111101-1100010-10110-1$ \\
$S_{5}$ & $-101-100111-11000-1101110-1010000-00$ \\
$S_{6}$ & $1100100-1-1-11-1011-10001010-11010000$ \\
$S_{7}$ & $100001-101010010001011-1-1-10-1100-11$ \\
$S_{8}$ & $0100-10-10110000-1-1100-11011-1110100$ \\
\hline
\end{tabular}

number of symbol repetitions within the preamble, $T_{\text {pri }}$ is the pulse repetition interval, $N_{\mathrm{s}}$ the total number of pulses per symbol and $E_{\mathrm{S}}$ denotes the symbol energy. Then, for the $i$ th basis symbol $\mathbf{S}_{i}$, the preamble symbol waveform $w_{i}(t)$ and the resulting preamble waveform $P_{i}(t)$ can be written as

$$
\begin{aligned}
& w_{i}(t)=\sqrt{\frac{E_{\mathrm{s}}}{N_{\mathrm{s}}}} \sum_{j=0}^{L-1} \mathbf{S}_{i}[j] \phi\left(t-j T_{\mathrm{pri}}\right), \\
& P_{i}(t)=\sum_{n=0}^{N_{\mathrm{sym}}-1} \mathbf{N}[n] w_{i}\left(t-n T_{\mathrm{sym}}\right),
\end{aligned}
$$

where $\mathbf{N}=\left[\begin{array}{lll}1 & 1 & \cdots\end{array}\right]_{1 \times N_{\mathrm{sym}}}$. Note that the pulse waveform coefficient vector for the entire preamble is then,

$$
\mathbf{P}_{i c}=\mathbf{N} \otimes \mathbf{S},
$$

where the operation $\otimes$ stands for the Kronecker product.

A receiver can process the received signal by either template matching (coherent) or energy detection (ED). Although coherent ranging gives better accuracy, the ED receiver offers advantages such as simplicity, operability at sub-Nyquist sampling rates (which determines the range resolution) and low cost. They are also more resilient to pulse shape distortion. In an ED receiver, a received signal is first passed through a low noise amplifier. It is then bandpass filtered and entered into a square-law device. The output of the square-law device is integrated over a time period $t_{s}$ and then sampled. The correlator output returns channel profile for each symbol repetition at the sampling resolution. If the channel is assumed to be stationary during the entire preamble, the correlator outputs of the repeated symbols can be coherently combined to improve the SNR. Note that probability of detecting the direct path increases with the SNR.

The standard adopts a two-way ranging protocol. Let RDEV represent a ranging capable device and RFRAME a frame that is tailored and used for ranging. In a so-called two-way timeof-arrival (TW-TOA) ranging protocol, the range between two RDEVs is determined via exchanging RFRAMEs and tracking their arrival times. The major advantage of this protocol is that it does not require time synchronization between ranging devices. Assume that RDEV A wants to perform ranging with RDEV B. Then, the sequence of events for the TW-TOA 


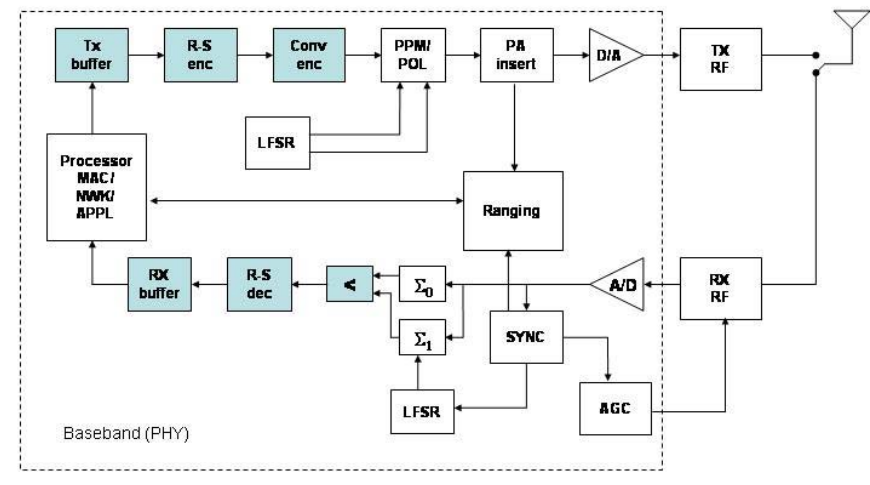

Fig. 2. Block diagram of a IR-UWB non-coherent transceiver

protocol would be as follows.

1) $R D E V$ A sends a range request frame, $R F R A M E_{\text {req }}$, to RDEV $B$, and records the time the frame departs from its antenna, $t=T_{1}$, according to its local clock.

2) $\mathrm{B}$ receives the $R F R A M E_{\text {req }}$ and sends back to $\mathrm{A}$ a range reply frame, $R F R A M E_{\text {rep }}$.

3) A records the time that it receives the $R F R A M E_{\text {rep }}$ at its antenna input according to its local clock. This time instant is denoted as $t=T_{2}$.

4) Finally, A calculates the difference between the two recorded times, $T_{r}=T_{2}-T_{1}$, and computes the range using the formula $r=c T_{r} / 2$, where $c$ is the speed of radio wave in the transmission medium.

5) By performing TW-TOA via at least three devices, A can compute its relative position with respect to them.

In reality, there is a non-zero processing delay at $\mathrm{B}$. Therefore, in the above algorithm $r$ would contain a positive bias. After incorporation of this processing delay, $T_{r}$ can be approximated as $T_{r}=2 T_{t}+T_{t a}^{B}$, where $T_{t}$ is the one-way time of flight of the RFRAME and $T_{t a}^{B}$ denotes the time elapsed at $\mathrm{RDEV} \mathrm{B}$ between its reception of the RFRAME req and its transmission of the $R F R A M E_{\text {rep }}$. It is commonly referred to as the turn-around time. $B$ should report $T_{t a}^{B}$ to $A$. The $R F R A M E_{\text {req }}$ can be piggy-backed by the time-stamp data.

\section{IMPLEMENTATION OF LOW COST NON-COHERENT TRANSCEIVER}

Our non-coherent radio prototype is designed using FPGAs and off-the-shelf RF components. Figure 2 shows some major functional blocks of the transceiver. The transceiver can be considered to consist of two sub-systems: the $R F$ front end and the baseband.

\section{A. RF front end}

There are three bands defined in the standard: $s u b-G H z$, low-band and high-band. The Sub-GHz band faces regulatory challenges in many countries. On the other hand, high-band incurs greater path loss which results in reduced range. Based on these considerations, we chose the low-band as the operating band for the prototype.

Some important parameters related to the RF circuit design are listed Table II. The standard defines three $500 \mathrm{MHz}$ channels in the low-band with the center frequencies of 3.494, 3.993 and $4.492 \mathrm{GHz}$ respectively. Our prototype operates at $3.4944 \mathrm{GHz}$.

\begin{tabular}{|c|c|c|}
\hline Parameter & Value & Note \\
\hline Band & $3.13-4.86 \mathrm{GHz}$ & low band \\
$f_{c 1}$ & $3.4944 \mathrm{GHz}$ & operating center frequency \\
$\Delta f$ & $\pm 20 \mathrm{ppm}$ & maximum frequency error \\
$B W_{3}$ & $499.2 \mathrm{MHz}$ & $-3 \mathrm{~dB}$ bandwidth \\
$P S D_{t x}$ & $-41.3 \mathrm{dBm} / \mathrm{MHz}$ & power spectrum density \\
$P_{t x}$ & $-14 \mathrm{dBm}$ & total transmitted power \\
\hline
\end{tabular}

TABLE II

RADIO FREQUENCY PARAMETERS

Most of the impulse generator RF circuits proposed recently focus on Gaussian monocycle pulses [8] [9] [10] [11]. These circuitry potentially can have higher efficiency but do not meet some or all of the following requirements for the 802.15.4a: high PRF (500 MHz), $500 \mathrm{MHz} 3 \mathrm{~dB}$ bandwidth and specific channelization spectrum mask and BPSK modulation (polarity scrambling). The conventional direct-conversion architecture, on the other hand, is well suited for the transmitter as shown in the top of Figure 3. The transmitter includes a low pass filter, a single mixer upconverter, RFLO generation circuit and necessary gain stages. A band pass filter is shared by both transmitter and receiver to reject the out of band emission or interference.

The 2 ns wide polarity modulated baseband pulse from the D/A is shaped with a low pass filter with $3 \mathrm{~dB}$ bandwidth of $\sim 250 \mathrm{MHz}$. In the standard, the baseband pulse shape is defined as having a minimum correlation with a root raised cosine pulse with specific pulse width and roll-off factor [2]. The energy detection based receiver is tolerant to pulse shape distortion. This allows the use of the low cost, efficient baseband LPFs for baseband pulse shaping providing the transmitted signal satisfies the spectrum mask. The pulse shaping filter is followed by the up-conversion mixer that converts baseband pulses to RF pulses with center frequency of $3.4944 \mathrm{GHz}$.

Our prototype is carefully designed to reduce carrier leakthrough. The leak-through problem is magnified in impulse radio due to the low duty cycle of the baseband pulse. We reduce the effect by good impedance match and balancing In addition, we can lower the RFLO power level when no pulse is transmitted. The low duty cycle also makes it possible to adjust the bias voltage dynamically to improve the power efficiency of the transmitter.

Both the RFLO clock and baseband clock $C L K_{B B}$ (499.2 $\mathrm{MHz})$ are generated from a free-running reference clock. The 


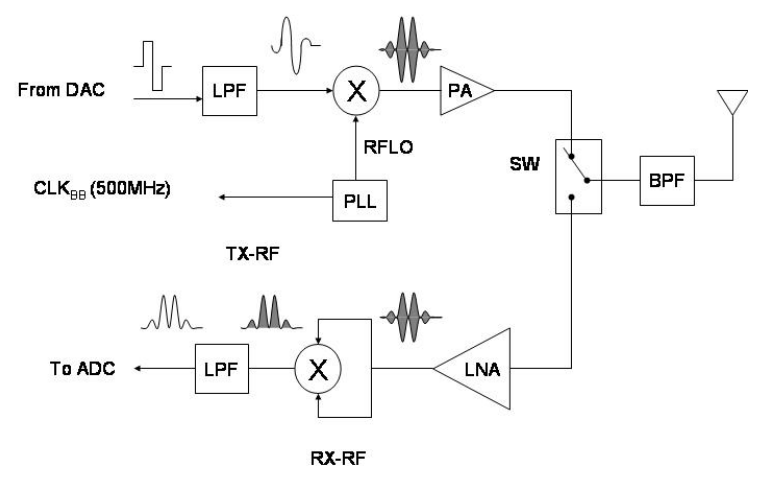

Fig. 3. Transmitter and receiver RF front end

standard specifies maximum carrier frequency error to be $\pm 20 \mathrm{ppm}$. The non-coherent data reception is insensitive to RF frequency offset; however, an accurate and stable baseband clock is needed for accurate ranging measurement.

The receiver RF front end consists of LNA, variable gain stages and an energy detection circuit as shown in Figure 3. To achieve ranging accuracy of $30 \mathrm{~cm}$, the energy detector circuit bandwidth needs to be $250 \mathrm{MHz}$. Commercially available low barrier Schottky diodes, commonly used as power detectors, have limited output bandwidth and can not be used for UWB signals. In our prototype design, a self-mixing circuit is used to perform the square-law operation. Such circuits meet the bandwidth requirement. The IF output is then filtered by a $250 \mathrm{MHz}$ baseband LPF.

The self mixing circuit requires matching phase to both inputs (RF and LO ports) of the mixer. Our simulation shows that with trace length mismatch of $5 \%$ of the carrier wavelength, the additional loss at the mixer output is less than $0.3 \mathrm{~dB}$. For $f_{c}=4 G H z$, the tolerance is $3.7 \mathrm{~mm}$ and manageable for PCB layout.

The noise figure (NF) requirement for the receiver circuit is mainly dictated by the high $E_{b} / N_{o}$ required for accurate ranging. It has been shown in [5] $E_{b} / N_{o} \geq 13 d B$ is needed to achieve $30 \mathrm{~cm}$ ranging accuracy, higher that the $E_{b} / N_{o}$ required for data communication. Our link budget estimation showed that the maximum NF of the receiver is $10 \mathrm{~dB}$. (Assuming 10 meter LOS path, 1024 symbols with length 31 ternary sequence and $\mathrm{PRF}$ of $4 \mathrm{MHz}, 0 \mathrm{~dB}$ antenna gain.)

Omni-directional antennas are used in our prototype. Even though the requirement for the antenna pattern is not as critical for ranging accuracy compared to other systems that rely on received signal strength for ranging, omni-directionality is needed for full connectivity since a node needs to be connected to at least 3 other nodes to be positioned in 2-D space and 4 nodes if 3-D positioning is required.

\section{B. Baseband Circuit}

The major functional blocks in the PHY layer are shown inside the dash-line box in Figure 2. Also shown in the box is a processor that runs MAC and upper layer software. The main hardware components in the baseband section include one FPGA, a high speed D/A and a high speed A/D. The FPGA we chose is a Xilinx V2Pro family device. Our MAC functions are implemented in one of the built-in PowerPCs.

As shown in Figure 2, the transmitter includes a ReedSolomon encoder, a convolutional encoder, BP-PPM modulation blocks and a preamble generation. The convolutional encoder and BPSK modulation do not have an impact on the performance of the noncoherent receiver, but are necessary for 15.4a standards compliance and spectrum smoothing.

The transmitter baseband only outputs 3 values $(+1,0$ and $-1)$, therefore, it only needs a simple 1.5 bit D/A converter. Such conversion circuits are readily available at low cost and high power efficiency. The receiver A/D converter requires minimum of 4-bits and sampling rate of $499.2 \mathrm{MHz}$. This high sampling rate enables us to achieve $30 \mathrm{~cm}$ ranging resolution. For non-ranging capable devices, lower sampling rates can be used if an integration circuit is implemented in the analog domain. This will lower the power consumption and reduce cost.

Inside the SYNC block in Figure 2 is a 31-tap correlator that si shared by the acquisition/synchronization (ACQ/SYNC) block and the ranging block. When the correlator outputs exceed the threshold, it activates the synchronization state machine which starts the synchronization process. The ACQ/SYNC willdetect and lock to the strongest path based on the first few symbols. Fast acquisition/synchronization is important to the ranging performance as the number of symbols available for ranging reduces if the synchronization is slow; this in turn lowers the processing gain in the ranging block.

Once the start of frame delimiter (SFD) is detected, the receiver begins data demodulation. For non-coherent receivers, the data is decoded by comparing the total energy in the two different burst slots. The window size for the energy collection is adjusted by the transmitted burst duration plus the channel spread. The output of the PPM demodulator is fed into the Reed-Solomon decoder. The decoded data are buffered for MAC software to read.

It is important to point out that even though at $A / D$ and D/A the data are sampled at $500 \mathrm{MHz}$, not all the blocks in the baseband need to run at this speed. All the shaded blocks in Figure 2 can operate at a much lower speed $(2 \mathrm{MHz})$. Taking advantage of this can drastically reduce the power consumption and design complexity.

1) Ranging Signal Processing: On the initiator side, the ranging block is responsible for registering the time elapsed from the end of the ranging request packet to the reception of the response packet. On the target side, it computes the time between the reception of the preamble and the start of the transmission of the response packet. Several counters are implemented in the block for this purpose. These counters are controlled directly by hardware but are accessible to the processor.

The correct detection of the first arrival of the pulse is critical in the ranging accuracy in a multi-path environment. 


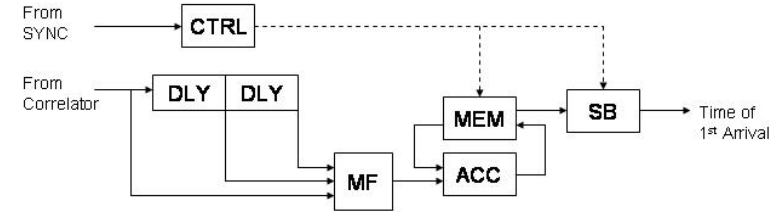

Fig. 4. Ranging Block Structure

Among all the algorithms proposed to deal with this issue, the algorithm proposed in [7] offers good performance in low $E_{b} / N_{o}$ environments and the presence of multi-user interference. The receiver first converts the received preamble to a 2-D energy matrix and runs it through a non-linear filter. It then averages the samples of each row and then performs a search back to locate the first arrival.

At first glance, it appears that implementing this algorithm would demand huge amount of resource since it performs matrix conversion and filtering over the entire preamble section. For a preamble with 1024 symbols and average PRF of $4 \mathrm{MHz}$, at $500 \mathrm{MHz}$ sample rate, this would require 2 million samples to be stored. In addition, the median filtering and averaging operation over the entire preamble is energy-demanding and time consuming. However, this ranging algorithm can be implemented very efficiently in hardware with low area and power. Figure 4 shows the actual implementation of the algorithm. The output of the correlator in the SYNC block is fed into the median filter along with two samples that are delayed by 1 and 2 symbol durations. For energy efficiency, the delay blocks are implemented using 3 dual-port RAM blocks with incrementing read and write address. The output of the median filter is fed into an accumulation block, which sums up the samples from different symbols. Once the end of the preamble is detected by the SYNC block, the search back is enabled to search for the first arrival and report its location. In stead of searching over the whole duration of the symbol, we reduced the search window to $256 \mathrm{~ns}$ which is longer than the channel spread under different models. This further reduces the total memory size as well as the number of operations.

\section{MAC and upper layer software}

The 802.15.4a standard uses simple Aloha as the multiple access protocol. Although not as efficient as other more sophisticated protocols, it is lightweight and can run on some low cost processors. In the prototype, we implemented the MAC and some simple network protocols in one of the PowerPCs inside the FPGA with 32KByte On-chip RAM and 128KByte ROM. Other peripherals required for the MAC processors include timers, interrupt controller and some interface and debug modules.

In our positioning demonstration system, the positioning solver is running on a host PC attached to one of the nodes through USB. It is possible to move the position solver to the node as the code size is small.

\section{EXPERIMENTAL RESUlTS}

The entire baseband fits in a single Xilinx X2VP30. Table III breaks down the resources used by transmitter, receiver (excluding ranging), ranging and the processor. All the software is running on a PowerPC. However, it is possible to run the software on a less powerful processor.

\begin{tabular}{|c|c|c|c|c|}
\hline Name & LUT & FF & Mem(Kb) & Proc \\
\hline TX PHY & 1282 & 1993 & 288 & 0 \\
RX PHY & 1621 & 2259 & 320 & 0 \\
RANGE & 1040 & 1279 & 64 & 0 \\
MAC PROC & 1691 & 1284 & 1280 & 1 \\
\hline
\end{tabular}

TABLE III

RADIO FREQUENCY PARAMETERS

Figure 5 and 6 show the captured waveforms of the preamble (single pulse) and data burst at transmitter antenna port, receiver mixer output and receiver LPF output. Figure 7 shows the spectrum of the transmitted signals. As shown in the figure, the power density is under $-41 \mathrm{dBm} / \mathrm{MHz}$ and the bandwidth is over $500 \mathrm{MHz}$, fulfilling the requirements of frequency regulators. We do however, observe the carrier leakthrough as shown in the figure. We expect improvement if we install the circuit that switches off the RFLO when no pulse is transmitted.

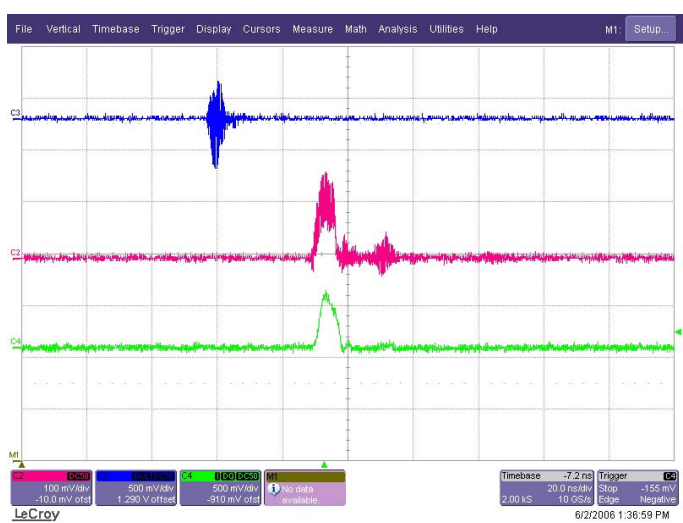

Fig. 5. Signal Waveform - Preamble Section (timebase:20ns/div)

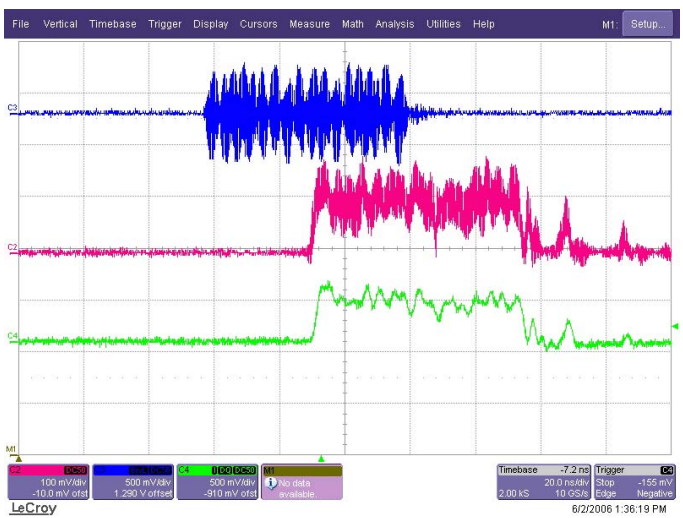

Fig. 6. Signal Waveform - Data Section (timebase:20ns/div) 


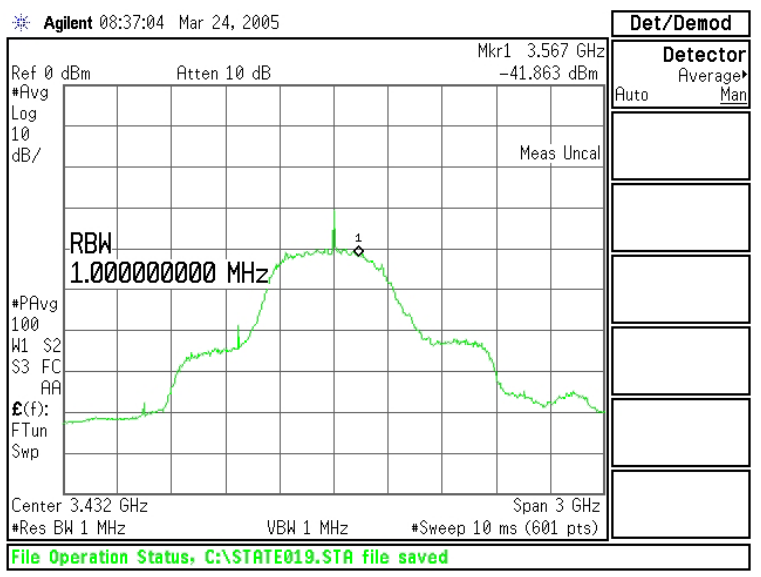

Fig. 7. Power Spectrum at Transmitter Antenna Port

The pair-wise data communication and ranging were first tested. Our experiment showed that with LOS, we can achieve slightly over 10 meter range while maintaining high ranging accuracy. By averaging 10 ranging measurements, we were able to achieve accuracy of $15 \mathrm{~cm}$. This matches the theoretical ranging limit. We observed that the accuracy remains almost unchanged while the two nodes under test are within the $10 \mathrm{~m}$ range. However, as the distance increases, the number of lost packets increases, which results in increased ranging-response time-outs. This indicates that the acquisition/synchronization logic is the limiting factor for the system range. It is possible to improve the system performance with more sophisticated synchronization algorithms.

Another observation we made during the test was that when the AGC is disabled, the ranging error increases when two nodes are very close to each other (less than 1 meter). Our investigation showed that this is caused by peak compression in the receiver path. When the received pulse is too strong, the peak of the received pulse is compressed. As our search-back algorithm uses the energy of the strongest pulse to determine the threshold for the first arrival pulse, the compression of peak value effectively lowers the threshold. As a result, the receiver sometimes erroneously identifies a noise peak as the earliest arriving path.

For positioning demonstration, we used the setup as shown in Figure 8. Five identical nodes are used in the network. One of the nodes is designated as the target node, the other 4 nodes are reference nodes. The reference nodes are stationary and their locations are known to the system. The target node is mobile and once requested by the system, it will exchange ranging packets $\left(r p_{n}\right.$ indicates the range probe to reference node $R_{n}, r a_{n}$ is the range ack packet from reference node $R_{n}$ ) with all the neighboring nodes. The target node computes the distance to all the reference nodes and then sends all the range data in one packet to one of the reference node which has a host PC attached to it. The position of the target node is computed and displayed on the host PC.

Without using any complex post-processing of the ranging data, our experimental system was able to track the target device with an average error of better than $\pm 30 \mathrm{~cm}$. The system is configured to update the position of the target 10 times per second. We also tried reducing the number of reference nodes to three and did not observer any degradation in accuracy.

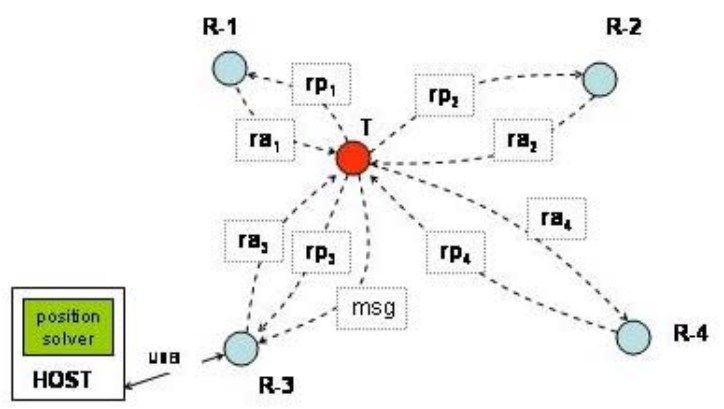

Fig. 8. Positioning demo system configuration

\section{COnClusion And Future Works}

As a low cost, low power option, the non-coherent devices are attractive in many applications. This paper presents a noncoherent radio design and prototype system we developed. We addressed some implementation issues and showed that a noncoherent transceiver can be implemented with low complexity and low power consumption. We tested our prototype system for data communication and ranging capability and the results showed that the ranging accuracy matches to the theoretical limit. Using these prototype radios, we also demonstrated submeter positioning accuracy.

\section{REFERENCES}

[1] IEEE 802.15 Task Group 4a website, "http://www.ieee802.org/Tg4a.html" [2] IEEE P802.15.4a/D7, January 2007, PART 15.4:Wireless Medium Access Control (MAC) and Physical Layer (PHY) Specifications for Low-Rate Wireless Personal Area Networks (LRWPANs)

[3] A.F.Molisch et al, "UWB-based Sensor Networks and the IEEE 802.15.4a Standard", International Conference Communications and Networking in China, pp. 1-6, October 2006

[4] S. Gezici et al, "Localization via ultra-wideband radios," IEEE Signal Processing Magazine, vol. 22, issue 4, pp. 70-84, July 2005.

[5] Z. Sahinoglu and S. Gezici, "Ranging for IEEE 802.15.4a ultra-wideband devices," IEEE Wireless and Microwave Technology Conference, Dec. 2006

[6] P. Orlik, S. Zhao and A.F. Molisch, "A Hybrid UWB Modulation Design Compatible for Both Coherent and Transmit-Reference Receivers", IEEE International Conference on Communications, June 2006

[7] Z. Sahinoglu and I. Guvenc, "Multiuser Interference Mitigation in Noncoherent UWB Ranging via Nonlinear Filtering", EURASIP Journal on Wireless Communications and Networking, Volume 2006, Article ID 56849, June 2006

[8] Sumit Bagga et al, "Codesign of an Impulse Generator and Miniaturized Antennas for IR-UWB", IEEE Transactions on microwave theory and techniques, Vol. 54, No. 4, April 2006, pp 1656-1666

[9] Youngkyun Jeong, Sungyong Jung, Jin Liu, "A CMOS Impulse Generator for UWB Wireless Communication Systems", Proc. of International Symposium on Circuits and Systems, 2004, pp 129-132

[10] Jochen Dederer, Christoph Schick, Andreas Trasser and Hermann Schumacher, "A SiGe impulse generator for single-band ultra-wideband applications" http://www.iop.org/EJ/abstract/0268-1242/22/1/S47/

[11] X. Chen and S. Kiaei, Monocycle shapes for ultra wide-band system Proc. of Int. Symp. Circuits and Systems, 2002, pp. 2629. 